\title{
The Reasonable Ineffectiveness of Mathematics
}

\author{
BY DEREK ABBOTT \\ School of Electrical and Electronic Engineering \\ The University of Adelaide, Adelaide, S.A. 5005, Australia
}
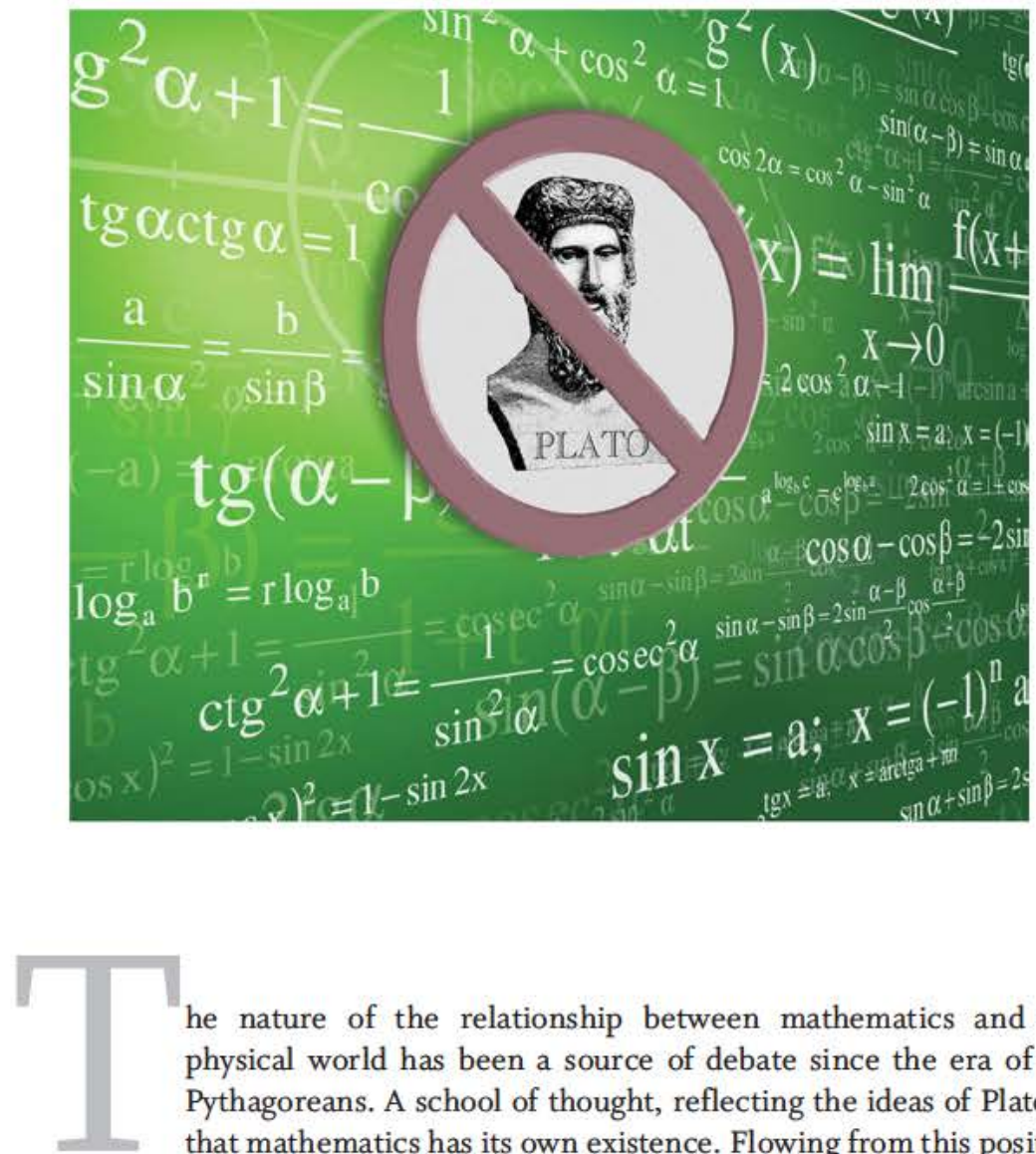

he nature of the relationship between mathematics and the physical world has been a source of debate since the era of the Pythagoreans. A school of thought, reflecting the ideas of Plato, is that mathematics has its own existence. Flowing from this position is the notion that mathematical forms underpin the physical universe and are out there waiting to be discovered.

The opposing viewpoint is that mathematical forms are objects of our human imagination and we make them up as we go along, tailoring them to describe reality. In 1921, this view led Einstein to wonder, "How can it be that mathematics, being after all a product of human thought which is independent of experience, is so admirably appropriate to the objects of reality?" [1].

In 1959, Eugene Wigner coined the phrase "the unreasonable effectiveness of mathematics" to describe this "miracle," conceding that it was something he could not fathom [2]. The mathematician Richard W. Hamming, whose work has been profoundly influential in the areas of computer science and electronic engineering, revisited this very question in 1980 [3].
Hamming raised four interesting propositions that he believed fell short of providing a conclusive explanation [3]. Thus, like Wigner before him, Hamming resigned himself to the idea that mathematics is unreasonably effective. These four points are: 1 ) we see what we look for; 2 ) we select the kind of mathematics we look for; 3) science in fact answers comparatively few problems; and 4) the evolution of man provided the model.

In this article, we will question the presupposition that mathematics is as effective as claimed and thus remove the quandary of Wigner's "miracle," leading to a non-Platonist viewpoint. ${ }^{1}$ We will also revisit Hamming's four propositions and show how they may indeed largely explain that there is no miracle, given a reduced level of mathematical effectiveness.

The reader will be asked for a moment of indulgence, where we will push these ideas to the extreme, extending them to all physical law and models. Are they all truly reified? We will question their absolute reality and ask the question: Have we, in some sense, generated a partly anthropocentric physical and mathematical framework of the world around us?

Why should we care? Among scientists and engineers, there are those that worry about such questions and there are those that prefer to "shut up and calculate." We will attempt to explain why there might be a

${ }^{1}$ This explains the inverted title of the present article, "The reasonable ineffectiveness of mathematics." 
useful payoff in resolving our philosophical qualms and how this might assist our future calculations.

\section{MATHEMATICIANS, PHYSICISTS, AND ENGINEERS}

The following is anecdotal and is by no means a scientific survey. However, in my experience of interacting with mathematicians, physicists, and engineers, I would estimate that about $80 \%$ of mathematicians lean to a Platonist view. ${ }^{2}$ Physicists, on the other hand, tend to be closeted non-Platonists. An ensemble of physicists will often appear Platonist in public, but when pressed in private I can often extract a non-Platonist confession.

Engineers by and large are openly non-Platonist. Why is that? Focusing on electrical and electronic engineering, as a key example, the engineer is well acquainted with the art of approximation. An engineer is trained to be aware of the frailty of each model and its limits when it breaks down. For example, we know that lumped circuit models are only good for low frequencies.

An engineer is also fully aware of the artificial contrivance in many models. For example, an equivalent circuit only models the inputs and outputs of a circuit, and ignores all the internal details. Moreover, the engineer knows the conditions under which these simplifications can be exploited.

An engineer often has control over his or her "universe" in that if a simple linear model does not work, the engineer, in many cases, can force a widget, by design, to operate within a restricted linear region. Thus, where an engineer cannot approximate linearity, he often linearizes by fiat.

A mathematical Platonist will often argue that number $\pi$ is a real entity, claiming that a geometric circle is a reified construct that exists inde-

\footnotetext{
${ }^{2}$ The interested reader is referred to [4] for an entertaining view of the non-Platonist position, and [5] for a Plationist perspective.
}

pendently of the universe. An engineer, on the other hand, has no difficulty in seeing that there is no such thing as a perfect circle anywhere in the physical universe, and thus $\pi$ is merely a useful mental construct.

In addition to the circle, many other ideal mathematical forms such as delta functions, step functions, sinusoids, etc., are in an engineer's mathematical toolbox and used on a daily basis. Like the circle, the engineer sees delta functions, and for that matter all functions, as idealities that do not exist in the universe. Yet, they are useful for making sufficiently accurate, yet approximate, predictions.

A physicist may have nightmares on studying a standard electronic engineering text, finding the use of negative time in the theory of noncausal filters. However, a non-Platonist engineer has no qualms about such transformations into negative spaces, as there is no ultimate reality there. These are all mental constructs and are dealt with in a utilitarian way, producing the results required for system design.

Hamming's paper marvels on how complex numbers so naturally crop up in many areas of physics and engineering, urging him to feel that "God made the universe out of complex numbers" [3]. However, for the engineer, the complex number is simply a convenience for describing rotations [7], and, of course, rotations are seen everywhere in our physical world. Thus, the ubiquity of complex numbers is not magical at all. As pointed out by Chappell et al. [8], Euler's remarkable formula $e^{j \pi}=-1$ is somewhat demystified once one realizes it merely states that a rotation by $\pi$ radians is simply a reflection or multiplication by -1 .

Engineers often use interesting mathematics in entirely nonphysical spaces. For example, the support vector machine (SVM) approach to classifying signals involves transforming physical data into nonphysical higher dimensional spaces and finding the optimal hyperplanes that separate the data. In telecommunications, coding theory can also exploit higher dimensional spaces [9]. In both these examples, physically useful outcomes result from entirely mental abstractions of which there are no analogs in the physical universe.

\section{DO FRACTALS HAVE THEIR OWN EXISTENCE?}

Roger Penrose, a mathematical Platonist, argues that a fractal pattern is proof of a mathematical entity having an existence of its own [6]. It is argued that the mathematician cannot foresee a beautiful fractal, before applying a simple iterative equation. Therefore, a fractal pattern is not a mental construct, but has its own existence on a Platonic plane waiting to be discovered.

A first objection is that there are an infinite number of ways to display the fractal data, and that to "see" a fractal we have to anthropocentrically display the data in the one way that looks appealing to our senses. Perhaps to an alien, a random pattern based on white noise might be more beautiful?

A second objection is that out of an infinite number of possible iterative equations, perhaps only negligible numbers of them result in fractal patterns and even fewer look appealing to humans. Take the analogy of a random sequence of digits. We know any infinite random sequence encodes all the works of Shakespeare and all the world's knowledge. If we preselect appealing parts of a random sequence, we have in fact cheated.

At the end of the day, a given set of rules that turns into an elegant fractal is really no different to, say, the set of rules that form the game of chess or that generate an interesting cellular automaton. The set of moves in a game of chess is evidently interesting and richly beautiful to us, but that beauty is no evidence that chess itself has a Platonic existence of its own. Clearly, the rules of chess are purely a contrived product of the human mind and not intrinsic to nature. 
A Platonist will argue that mathematical forms follow from a set of axioms, and thus exist independently of our knowledge of them. This situation is no different to our lack of foreknowledge of a fractal pattern, before exercising its originating equation. What can we say of the axioms themselves? I argue that they are also mental abstractions, and an example is given in Section $\mathrm{V}$ to illustrate that even the simple counting of objects has its physical limits. Thus, axioms based on the assumption of simple counting are not universally real.

\section{THE \\ INEFFECTIVENESS OF MATHEMATICS}

So far, we have argued that mathematics is a merely mental abstraction that serves useful purposes. A further response to answer Wigner's thought that the effectiveness of mathematics is a "miracle" is to suggest that this effectiveness might be overstated.

What we are finding in electronic engineering is that the way we mathematically model and describe our systems radically changes as we approach the nanoscale and beyond. In the 1970s, when transistor MOSFET lengths were of the order of micrometers, we were able to derive from physical first principles elegant analytical equations that described transistor behavior, enabling us to design working circuits. Today, we produce deep submicrometer transistors, and these analytical equations are no longer usable, as they are swamped with too many complicated higher order effects that can no longer be neglected at the small scale. Thus, in practice, we turn to empirical models that are embedded in today's computer simulation software for circuit design. Traditional analytical mathematics simply fails to describe the system in a compact form.

Another example is the use of Maxwell's equations for modeling integrated electromagnetic devices and structures. In modern devices, due to the complexity of design, we no long- er resort to analytical calculations; instead, electromagnetic simulation programs that use numerical methods are now the standard approach.

The point here is that when we carry out engineering in different circumstances, the way we perform mathematics changes. Often the reality is that when analytical methods become too complex, we simply resort to empirical models and simulations.

The Platonist will point out that the inverse square law for gravitation is spectacularly accurate at predicting the behavior of nearby planets and distant stars across vast scales. However, is that not a self-selected case conditioned on our human fascination with a squared number? Furthermore, due to inherent stochasticity in any physical system, at the end of the day, we can only ever experimentally verify the square law to within a certain accuracy. While the Newtonian view of gravitation is a spectacularly successful model, it does not hold what we believe to be the underlying reality; it has been surpassed by the 4-D curved spaces of general relativity, and this is now the dominant viewpoint until a better theory comes along.

Note that mathematics has lesser success in describing biological systems, and even less in describing economic and social systems. But these systems have come into being and are contained within our physical universe. Could it be they are harder to model simply because they adapt and change on human time scales, and so the search for useful invariant properties is more challenging? Could it be that the inanimate universe itself is no different, but happens to operate on a timescale so large that in our anthropocentrism we see the illusion of invariance?

An energy-harvesting device that is in thermal equilibrium cannot extract net energy or work from its environment. However, if we imagine that human lifespans are now reduced to the timescale of one thermal fluctuation, the device now has the illusion of performing work. We experience the Sun as an energy source for our planet, partly because its lifespan is much longer than human scales. If the human lifespan were as long as the universe itself, perhaps our sun would appear to be short-lived fluctuation that rapidly brings our planet into thermal equilibrium with itself as it "blasts" into a red giant. These extreme examples show how our anthropocentric scales possibly affect how we model our physical environment.

\section{A. Hamming's First Proposition: We See What We Look For}

Hamming suggests here that we approach problems with a certain intellectual apparatus, and, thus, we anthropocentrically select out that which we can apply our tools to [3]. Our focus shifts as new tools become available. In recent years, with the emerging paradigms of complex systems and mining of so-called big data, traditional mathematics has a smaller role and large brute force computing is used to search for the patterns we are looking for.

\section{B. Hamming's Second \\ Proposition: We Select the Kind of Mathematics We Look For}

Here, Hamming points out that we tailor mathematics to the problem at hand [3]. A given set of mathematical tools for one problem does not necessarily work for another. The history of mathematics shows a continual development; for example, scalars came first, then we developed vectors, then tensors, and so on. So as fast as mathematics falls short, we invent new mathematics to fill the gap.

By contrast, a Platonist will argue for the innateness of mathematics by pointing out that we sometimes invent useful mathematics before it is needed. For example, Minkowski and Riemann developed the theory of 4-D curved spaces in the abstract, before Einstein found it of utility for general relativity. I argue that this innateness is illusory, as we have cherry picked a successful coincidence from a 
backdrop of many more cases that are not as fortuitous.

\section{Hamming's Third Proposition: Science Answers Comparatively Few Problems}

Taking into account the entire human experience, the number of questions that are tractable with science and mathematics are only a small fraction of all the possible questions we can ask. Gödel's theorem also set limits on how much we can actually prove. Mathematics can appear to have the illusion of success if we are preselecting the subset of problems for which we have found a way to apply mathematics.

A case in point is the dominance of linear systems. Impressive progress has been made with linear systems, because the ability to invoke the principle of superposition results in elegant mathematical tractability. On the other hand, developments in nonlinear systems have been arduous and much less successful. If we focus our attention on linear systems, then we have preselected the subset of problems where mathematics is highly successful. $^{3}$

\section{Hamming's Fourth Proposition: The Evolution of Man Provided the Model}

A possibility is that the quest for survival has selected those who are able to follow chains of reasoning to understand local reality. This implies that the intellectual apparatus we use is in some way already appropriate. Hamming points out that, to some extent, we know that we are better adapted to analyzing the world at our human scale, given that we appear to have the greatest difficulties in reasoning about the very small scale and the very large scale aspects of our universe.

\footnotetext{
${ }^{3}$ One might remark that many fundamental processes rather successfully approximate linear models, and this may again seem like Wigner's magic. However, is this not self-referential? What we humans regard as "fundamental" tend to be those things that appear linear in the first place.
}

\section{E. Physical Models as a Compression of Nature}

There is a fifth point we might add to Hamming's four propositions, and that is that all physical laws and mathematical expressions of those laws are a compression or compact representation. They are necessarily compressed due to the limitations of the human mind. Therefore, they are compressed in a manner suited to the human intellect. The real world is inherently noisy and has a stochastic component, so physical models are idealizations with the rough edges removed.

Thus, when we "uncompress" a set of equations, to solve a given problem, we will obtain an idealized result that will not entirely match reality. This can be thought of as uncompressing a video that was initially subjected to lossy compression. There will always be lossy information leakage but, provided the effects we have neglected are small, our results will be useful.

\section{F. Darwinian Struggle for the Survival of Ideas}

A sixth point we can add to Hamming's list is that Wigner's sense of "magic" can be exorcised if we see that the class of successful mathematical models is preselected. Consider the millions of failed models in the minds of researchers, over the ages, which never made it on paper because they were wrong. We tend to publish the ones that have survived some level of experimental vindication. Thus, this Darwinian selection process results in the illusion of automatic success; our successful models are merely selected out from many more failed ones.

Take the analogy of a passenger on a train, pulling the emergency stop lever, saving the life of a person on a railway track; this seems like a miracle. However, there is no miracle once we look at the prior that many more people have randomly stopped trains on other occasions saving no lives. A genius is merely one who has a great idea, but has the common sense to keep quiet about his other thousand insane thoughts.

\section{WHAT ABOUT THE ALIENS?}

Mathematical Platonists often point out that a hypothetical alien civilization will most likely discover the number $\pi$ and put it to good use in their alien mathematics. This is used to argue that $\pi$ has its own Platonic existence, given that it is "out there" for any alien to independently discover.

Do aliens necessarily know number $\pi$ ? Do aliens even have the same view of physics?

Given the simplicity of geometric objects such as ideal circles and squares, an alien race may indeed easily visualize them. However, this is not true of all our mathematical objects, especially for those with increased complexity. For example, an alien race may never find the Mandlebrot set, and may not even pause to find it interesting if found by chance.

An alien race might happily do all its physics and engineering without the invention of a delta function. Perhaps the aliens have parameterized all their physical variables in a clever way, and if we were to compare we would find that one of our variables was surprisingly redundant.

Perhaps not all aliens have a taste for idealizations, nor Occam's razor. Maybe all their physical equations are stochastic in nature, thereby realistically modeling all physical phenomena with inherent noise.

One might also hypothesize a superintelligent alien race with no need for long chains of analytical mathematical reasoning. Perhaps their brains are so powerful that they jump straight into performing vast numerical simulations, based on empirical models, in their heads. So the question of the effectiveness of mathematics, as we know it, has no meaning for them. This thought experiment also illustrates that human mathematics serves us to provide the necessary compression of representation required by our limited brain power. 
V. ONE BANANA, TWO BANANA, THREE BANANA, FOUR

I deeply share Hamming's amazement at the abstraction of integers for counting [3]. Observing that six sheep plus seven sheep make 13 sheep is something I do not take for granted either.

A deceptively simple example to illustrate the limitations in the correspondence between the ideal mathematical world and reality is to dissect the idea of simple counting. Imagine counting a sequence of, say, bananas. When does one banana end and the next banana begin? We think we know visually, but to formally define it requires an arbitrary decision of what minimum density of banana molecules we must detect to say we have no banana.

To illustrate this to its logical extreme, imagine a hypothetical world where humans are not solid but gaseous and live in the clouds. Surely, if we evolved in such an environment, our mathematics would not so readily encompass the integers? This relates to Hamming's Fourth Proposition, where our evolution has played a role in the mathematics we have chosen.

Consider the physical limits when counting a very large number of bananas. Imagine we want to experimentally verify the 1-to-1 correspondence between the integer number line, for large $N$, with a sequence of physical bananas. We can count bananas, but for very large $N$, we need memory to store that number and keep incrementing it. Any physical memory will always be subject to bit errors and noise, and, therefore, there are real physical limits to counting.

An absolute physical limit is when $N$ is so large that the gravitational pull of all the bananas draws them into a black hole. ${ }^{4}$ Thus, the integer number line is lacking in absolute reality. Davies goes a step further and argues that real numbers are also a fiction; they cannot be reified as the universe

\footnotetext{
${ }^{4}$ It is of interest to note here that Lloyd has exploited black holes to explore the physical limits of computation [10].
}

can store at most $10^{122}$ bits of information [11].

\section{STRONG NON-PLATONISM}

For the purposes of this essay, we have loosely labeled mathematical Platonism as the position that ideal mathematical objects exist and they are waiting to be discovered. Similarly, physical laws are also reified.

What we loosely refer to as nonPlatonism is the view that mathematics is a product of human imagination and that all our physical laws are imperfect. Nature is what it is, and by physical law we are, of course, referring to man's compression of nature.

The reader is now asked to entertain strong non-Platonism, where all physical laws are tainted with anthropocentrism and all physical models have no real interpretative value. The interpretive value of physics is purely illusory. After all, a beam of light passing through a slit knows nothing of Fourier transforms; that is an overlaid human construct.

Imagine 3-D particles passing through a 2-D universe. A 2-D flatlander [12] can create beautiful interpretations, which may even have some predictive accuracy, regarding these mysterious particles that appear, change size, and then disappear. But these interpretations are to some extent illusory and at best incomplete.

In our world, we are trapped on human length scales, human power scales, and human time scales. We have created clever instruments that extend our reach, but we are hopelessly lacking in omnipotence.

In some cases, we knowingly build up a set of models with imaginary interpretative value purely for convenience. For example, we can measure the effective mass and drift velocity of holes in a semiconductor, knowing fully well that semiconductor holes are an imaginary artifice. We exploit them as a mental device because they provide a shortcut to giving us predictive equations with which we can engineer devices.
John von Neumann stated all this more succinctly: "The sciences do not try to explain, they hardly even try to interpret, they mainly make models. By a model is meant a mathematical construct which, with the addition of certain verbal interpretations, describes observed phenomena. The justification of such a mathematical construct is solely and precisely that it is expected to work" [13].

\section{IMMUTABILITY}

Another way to see the potential frailty of physical "laws" created by man is to ask which principles in physics are sacred and immutable? I will leave this as an exercise for the reader. However, when I tried the thought experiment I was able to stretch my imagination to permitting a violation of everything we know. At some vast or small scale of any set of parameters, one can imagine breakdowns in the laws, as we know them.

Is there anything we can hold onto as inviolate under any circumstances? What about Occam's razor? I would like to hold onto Occam's razor as immutable, but I fear that it too may be embedded with anthropocentrism. When classifying physical data, it is known that God does not always shave with Occam's razor [14]. Could it be that, as the human brain demands a compression of nature, Occam's razor is our mental tool for sifting out compact representations?

\section{A PERSONAL STORY}

As this is an opinion piece, it might be pertinent to understand where my opinions come from. I have a distinct memory of being alone playing on the floor, at the age of four, with a large number of cardboard boxes strewn across the room. I counted the boxes. Then, I counted them again and obtained a different number. I repeated this a few times obtaining different numbers. This excited me because I thought it was magic and that boxes were appearing and disappearing. But the magic unfortunately disappeared 
and I eventually kept obtaining a run of the same number. In a few minutes I concluded that my initial counting was inaccurate and that there sadly never was any magic. This was my first self-taught lesson in experimental repeatability and the removal of magic from science.

At both elementary school and high school, mathematics was my favorite subject, although I spent far too many years worrying about the concept of infinity. Taking a limit to infinity was something I simply got used to, minus the desire to wildly embrace it. I struggled with accepting negative numbers, and raising numbers to the power of zero seemed absurd. ${ }^{5}$ I remember a great sense of disappointment when I was told that vectors could not be divided. Something was not quite right, but then I could not put my finger on it. After all complex numbers contain a direction and magnitude, yet can be divided. The more mathematics I learned the more it seemed like an artificial hodgepodge of disparate tools, rather than a divine order.

While I loved the beauty of mathematical proofs and the search for them, it worried me that each proof needed creative ad hoc handcrafting; there was no heavenly recipe book. The nature of proofs began to appear philosophically suspect to me, for example, how do we really know if a proof is correct if it is too long? A mathematical proof is the demonstration that a proposition is correct with a level of certainty that two mathematicians somewhere in the world understand it; that was in jest, of course, but the proof of Fermat's last theorem is arguably close to pushing that boundary.

At the age of 19, in my undergraduate university library, I stumbled on a textbook that changed my life. In its introduction, it stated that mathematics is a product of the human mind. Obviously, all my teachers must have

\footnotetext{
${ }^{5}$ In retrospect, I am astonished with how my mindset was so 16th century. I will argue that it is the ravages of Platonism that can lock us into that mold.
}

been mathematical Platonists, as I had never heard such an outlandish statement before. Immediately, a great burden lifted from my shoulders, and my conversion to non-Platonism was instant. This was a road to Damascus experience for me, and my philosophical difficulties that haunted me vanished.

As Hamming aptly states, "The postulates of mathematics were not on the stone tablets that Moses brought down from Mt. Sinai” [3].

\section{WHY NOT JUST SHUT UP AND CALCULATE?}

Why should we care about the nature of mathematics? My personal story for one illustrates that there is greater freedom of thought, once we realize that mathematics is something we entirely invent as we go along. This view can move us ahead and free us from an intellectual straight jacket. With the shackles removed, we can proactively manipulate, improve, and apply mathematics at a greater rate.

If we discard the notion that mathematics is passed down to us on stone tablets, we can be more daring with it and move into realms previously thought impossible. Imagine where we could be now if the centuries of debate over negative numbers could have been resolved earlier.

Another problem with mathematics today is the lack of uniformity in the tools we use. For example, we have the Cartesian plane and the Argand plane. They are isomorphic to each other, so why must we have both? We have complex numbers and quaternions. We have scalars, vectors, and tensors. Then, we have rather clunky dot and cross products, where the cross product does not generalize to higher dimensions.

It turns out to be something of a historical accident that the vector notation, with dot and cross products, was promoted by Gibbs and Heaviside, giving us a rather mixed bag of different mathematical objects.

Clifford's geometric algebra on the other hand, unifies all these mathe- matical forms [8], [15] [17]. It uses Cartestian axes and replaces complex numbers, quaternions, scalars, vectors, and tensors all with one mathematical object called the multivector. Dot and cross products are replaced with one single operation called the geometric product. This new type of product is elegant and follows the elementary rules for multiplying out brackets, with the extra rule that elements do not commute. You cannot divide traditional vectors, but multivectors do not have this restriction. All the properties naturally extend to higher dimensions, and thus the limitations of the cross product are overcome. This formalism is therefore simple and powerful, and delivers improved mathematical compression tailored for the limited human mind.

While this approach has existed since 1873 , it has been largely sidelined, as Gibbs and Heaviside favored dot and cross products. However, in physics, engineering, and computer science there is an emerging interest in reviving this mathematics due to its power and simplicity. To this end, we foreshadow a tutorial paper on geometric algebra for electrical and electronic engineers to be published in the Procendings of the IEEE at a later date [18].

\section{CONCLUSION}

Science is a modern form of alchemy that produces wealth by producing the understanding for enabling valuable products from base ingredients. Science is merely functional alchemy that has had a few incorrect assumptions fixed, but has in its arrogance replaced them with more insidious ones. The real world of nature has the uncanny habit of surprising us; it has always proven to be a lot stranger than we give it credit for.

Mathematics is a product of the imagination that sometimes works on simplified models of reality. Platonism is a viral form of philosophical reductionism that breaks apart holistic concepts into imaginary dualisms. I argue that lifting the veil of 
mathematical Platonism will accelerate progress. In summation, Platonic ideals do not exist; however, ad hoc elegant simplifications do exist and are of utility provided we remain aware of their limitations.

Mathematics is a human invention for describing patterns and regularities. It follows that mathematics is then a useful tool in describing regularities we see in the universe. The reality of the regularities and invariances, which we exploit, may be a little rubbery, but as long as they are sufficiently rigid on the scales of interest to humans, then it bestows a sense of order.

\section{Acknowledgment}

This paper is based on a talk the author presented at a workshop entitled The Nature of the Laws of Phy-

\section{REFERENCES}

[1] A. Einstein, Geometrie und Erfahrung. Berlin, Germany: Springer-Verlag, 1921.

[2] E. P. Wigner, "The unreasonable effectiveness of mathematics in the natural sciences," Commun. Pure Appl. Math., vol. XIII, pp. 1-14, 1960.

[3] R. W. Hamming, "The unreasonable effectiveness of mathematics.," Amer. Math. Monthly, vol. 87, no. 2, pp. 81-90, 1980.

[4] D. C. Stove, The Plato Cult and Other Philosophical Follies. Oxford, U.K.: Blackwell, 1991.

[5] S. C. Lovatt, New Skins for Old Wine: Plato's Wisdom for Today's World. Boca Raton, FL, USA: Universal, 2007.

[6] R. Penrose, The Road to Reality: A Complete Guide to the Laws of the Universe. New York, NY, USA: Knopf, 2004.

[7] E. O. Willoughby, "The operator $j$ and a demonstration that $\cos \theta+j \sin \theta=\mathrm{e}^{\mathrm{j} \theta}$, , in Proc. Inst. Radio Electron. Eng., vol. 26, no. 3, pp. 118-119, 1965. sics, December 17 19, 2008, Arizona State University (ASU), Phoenix, AZ, USA. The author would like to thank all the attendees that provided useful comments both for and against Platonism including: S. Aaronson of the Massachusetts Institute of Technology (MIT, Cambridge, MA, USA); P. C. W. Davies of ASU; G. F. R. Ellis of the University of Cape Town (Cape Town, South Africa); G. J. Chaitin of IBM (Armonk, NY, USA); A. J. Leggett of the University of Illinois at Urbana Champaign (UIUC, Urbana, IL, USA); N. D. Mermin of Cornell University (Ithaca, NY, USA); L. Susskind of Stanford University (Stanford, CA, USA); and S. Weinstein of the University of Waterloo (Waterloo, ON, Canada). The author is also grateful for a number of formative discussions on the topic, over the years, with C. Shalizi

[8] J. M. Chappell, A. Iqbal, and D. Abbott, Geometric Algebra: A Natural Representation of Three-Space. [Online]. Available: http:// arxiv.org/pdf/1101.3619.pdf

[9] M. El-Hajjar, O. Alamri, J. Wang, S. Zummo, and L. Hanzo, "Layered steered space-time codes using multi-dimensional sphere packing modulation," IEEE Trans. Wireless Commun., vol. 8, no. 7, pp. 3335-3340, Jul. 2009.

[10] S. Lloyd, "Ultimate physical limits to computation," Nature, vol. 406, no. 6799, pp. 1047-1054, 2000.

[11] P. C. W. Davies, The Goldilocks Enigma: Why Is the Universe Just Right for Life? London, U.K.: Penguin, 2007.

[12] E. A. Abbott, Flatland: A Romance of Many Dimensions. London, U.K.: Seeley, 1884.

[13] J. von Neumann, "Method in the physical sciences," in The Unity of Knowledge, L. Leery, Ed. New York, NY, USA: Doubleday, 1955, pp. 157-164. of Carnegie Mellon University (CMU, Pittsburgh, PA, USA) and P. C. W. Davies of ASU. A special thanks goes to A. J. Leggett of UIUC, for pointing out the problem of integer counting in the case of hypothetical gaseous beings - Tony was, in turn, inspired by Hawkins [19]. The author would also like to thank K. Wiesenfeld of Georgia Tech (Atlanta, GA, USA), for his thought experiment of an alien with computational mental powers sufficient to simulate the environment. The author asked a number of colleagues to proofread earlier drafts of this article, with strict instructions to be brutal. He is grateful to R. E. Bogner, J. M. Chappell, P. C. W. Davies, B. R. Davis, G. F. R. Ellis, M. D. McDonnell, A. P. Flitney, C. Mortensen, and W. F. Pickard for supplying gracious brutality in the spirit of debate.

[14] H. Bensusan, "God doesn't always shave with Occam's razor Learning when and how to prune," in Lecture Notes in Computer Science. Berlin, Germany: Springer-Verlag, 1998, vol. 1398, pp. 119-124.

[15] D. Hestenes, New Foundations for Classical Mechanics: Fundamental Theories of Physics. New York, NY, USA: Kluwer, 1999.

[16] S. Gull, A. Lasenby, and C. Doran, "Imaginary numbers are not real The geometric algebra of spacetime," Found. Phys., vol. 23, no. 9, pp. 1175-1201, 1993.

[17] M. Buchanan, "Geometric intuition," Nature Phys., vol. 7, no. 6, p. 442, 2011.

[18] J. M. Chappell, S. P. Drake, L. J. Gunn, A. Iqbal, A. Allison, and D. Abbott, "Geometric algebra for electrical and electronic engineers," in Proc. IEEE, 2014.

[19] D. Hawkins, The Language of Nature. San Francisco, CA, USA: Freeman, 1964. 